\title{
Primary Cutaneous Nocardiosis in a Heart Transplant Patient: A Case Report
}

\author{
Kiran Devaraj, MD, Joanna Rodriguez, MD, Bryan Hess, MD and Paul Mather, MD
}

\section{Case}

A 38-year-old female with history of longstanding non-ischemic cardiomyopathy underwent orthotopic heart transplantation (OHT). Her past medical history was significant for factor $\mathrm{V}$ leiden and methylenetetrahydrofolate reductase (MTHFR) heterozygous deficiencies with chronic pulmonary embolism, sickle cell trait, atrial flutter, type 2 diabetes mellitus, and hypertension. The patient had a long and complicated course post-transplantation. Immediately after OHT, she was noted to have donor- specific human leukocyte antigen (HLA) antibodies treated with 5 cycles of plasmapheresis. On further biopsies it was noted that she had acute cellular rejection requiring pulse-dose parenteral steroids on multiple occasions. Her immunosuppression therapy consisted of tacrolimus, mycophenolate and prednisone. Six months post-transplant she was noted to have a spontaneous $4 \mathrm{~cm}$ right calf muscle hematoma based on lower extremity ultrasound that was felt to be due to her underlying hematologic disease. At 7 months post-transplant the patient was hospitalized twice. The first admission was for multifocal necrotizing pneumonia. Although sputum cultures were non-diagnostic, the patient improved with 2 weeks of antibiotic treatment. The second admission occurred after a routine right heart catheterization that showed hemodynamic parameters concerning for rejection. At that time, the patient received empiric parenteral pulse steroids while waiting for final pathology results; these were negative for rejection and the patient's immunosupressants were continued at previous doses.

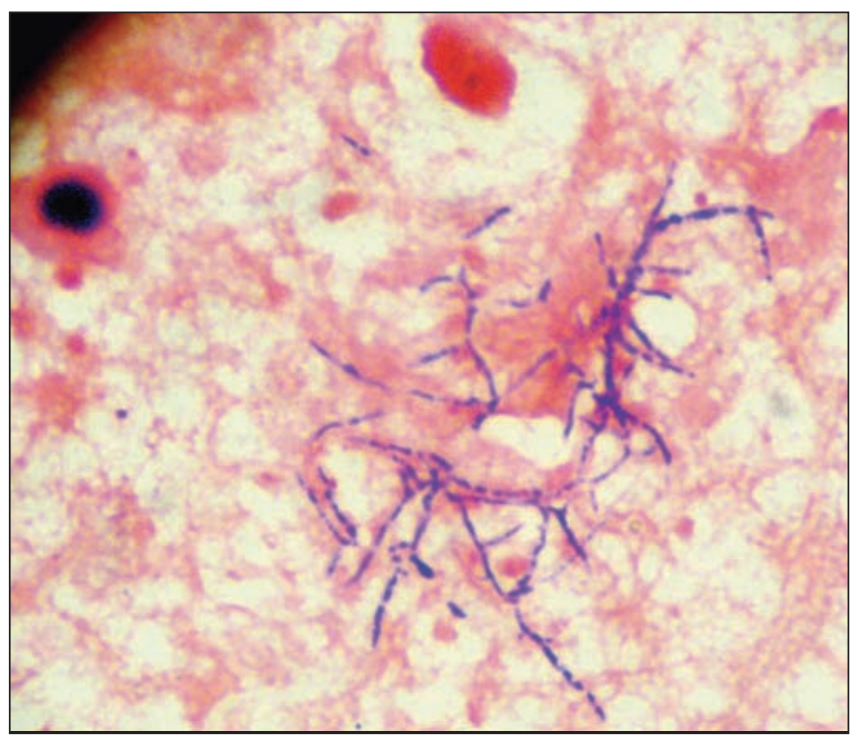

Figure 1. Gram stain from our patient showing gram positive bacilli bacilli.
The newest hospitalization was 8 months post-transplant, when she was admitted with a chief complaint of worsened right-sided calf pain for a month. On physical exam the patient was noticed to have an area of warmth, induration and palpable tenderness. Patient denied any other symptoms, and specifically denied fevers, chills, respiratory symptoms or trauma. Imaging studies on admission included a lower extremity ultrasound that showed the hematoma had increased in size to $9 \mathrm{~cm}$ from previous study 2 months prior. Lab studies to assess any acute hematologic cause of recurrent spontaneous hematoma were negative. The differential diagnosis on admission included a leg abscess; however the absence of fever, leukocytosis and loculations made it seem unlikely. A calf MRI performed 4 days later showed the collection had increased to $13 \mathrm{~cm}$. It was complex but still compatible with hematoma, although superimposed infection could not be ruled out. At this point it was decided for the patient to undergo an ultrasound guided fluid aspiration for diagnostic and therapeutic purpose, given the patient's continuing pain and rate of growth. Fluid aspiration yielded $50 \mathrm{cc}$ of pus. Broad spectrum antibiotics were started, and that evening the patient went to the operating room for an incision and drainage. The following day the wound culture grew a modified acid fast gram positive bacilli (by modified Kinyoun method) that immediately raised the concern for infection with nocardia species, especially given patient's immunosuppression and recent treatment with pulse-dose parenteral steroids. Patient's antibiotics were narrowed to intravenous ceftriaxone and patient was discharged home on this regimen, to convert to oral trimethoprimsulfamethoxazole (TMP-SMX) 4 weeks later for at least a 6

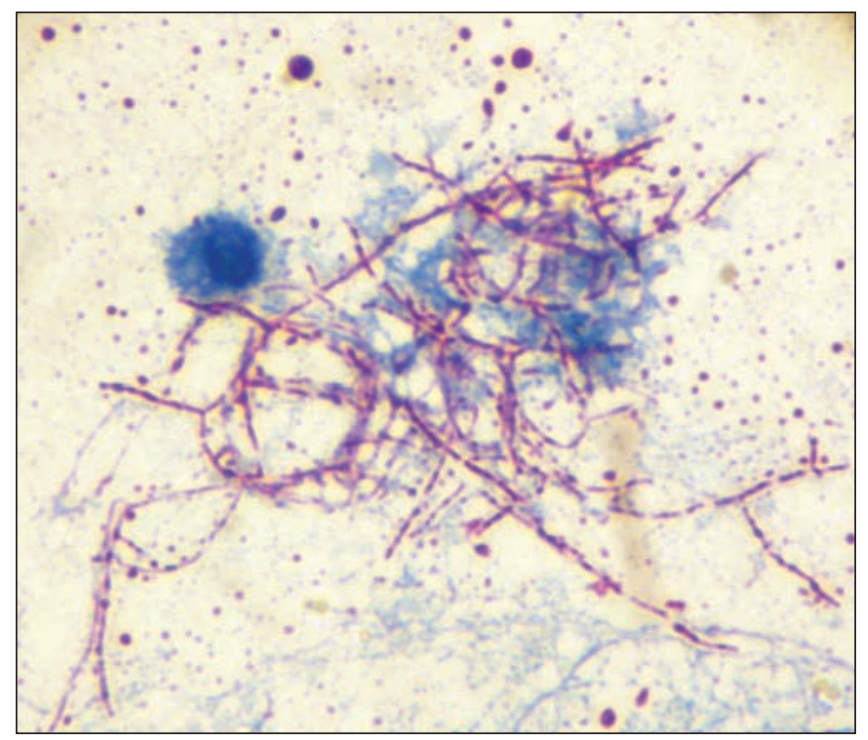

Figure 2. Modified Acid Fast (Kinyoun) Stain showing acid fast bacilli 
month course. Final DNA sequencing results of the organism confirmed the diagnosis of Nocardia cyriacigeorgica.

\section{Discussion}

Nocardia bacteria are weakly acid-fast (by modified Kinyoun, Ziehl-Neelsen, and Fite-Faraco methods) gram positive bacilli that grow slowly in aerobic culture. Nocardia species are saprophytes that are found worldwide in soil. There are various nocardia species that cause human disease, most commonly $N$. brasiliensis, $N$. otitidiscaviarum, $N$. farcinica, N. nova, $N$. transvalensis, and N. pseudobrasiliensis. ${ }^{1}$ Our patient's pathogen, $N$. cyriacogeorgica, is the most common cause of nocardiosis in the southern United States. ${ }^{2}$

Nocardia infections are rare among the general population of the United States, with an approximate incidence of 0.35-4 cases per 100000 people. ${ }^{3}$ Infections are concentrated to people with depressed cellular-mediated immunity, including patients with HIV, solid organ transplants, bone marrow transplants, cancer, and rheumatologic disease on immunosuppressive medications. In these patients, nocardia infections can have significant mortality, described as $11-67 \%$ (in case series with sample sizes of greater than or equal to 10$){ }^{4}$

Nocardia infections most commonly affect the lungs, central nervous system (CNS) system and skin. Nocardia pneumonia has a tendency for nodularity, cavitation and empyema. ${ }^{4}$ One half of patients present with extrapulmonary disease. CNS disease is most commonly an abscess. Therefore, patients with Nocardia skin infections may have primary cutaneous infection, or may have disseminated infection. Among immunocompetent persons, there are case reports of primary cutaneous infection. ${ }^{4}$

Primary nocardia skin and soft tissue infections are divided into three main categories: mycetomas, lymphocutaneous nocardiosis and primary cutaneous nocardiosis. Some form of traumatic skin opening classically precedes all three types, although iatrogenic cases have been reported. ${ }^{5}$ In tropical countries where mycetomas (Madura foot) are highly prevalent, nocardia is an alternative cause (after the more common fungal pathogens), resulting in non-painful chronic suppurative infection with draining sinuses and discharging granules. ${ }^{6}$ Primary cutaneous nocardiosis may include soft-tissue abscess, cellulitis, bulla or ulcer, but is classically acute and painful. ${ }^{6}$ Lymphocutaneous nocardiosis consists of an acute primary cellulitis with lymphangitic spread and causes lymphadenitis. ${ }^{7,8}$

Medical treatment of cutaneous nocardia infections is largely empiric and based on experience with treatment of invasive disease. Historically, oral TMP-SMX has been used for treatment of nocardiosis, with minocycline as an alternative. Given the high mortality of serious or invasive infections, an empiric regimen of TMP-SMX, amikacin, and ceftriaxone or imipenem is recommended. ${ }^{1,3}$ Of note, more recent data suggests growing TMP-SMX resistance. ${ }^{9}$ Treatment in our patient was difficult given her chronic kidney disease and nephrotoxic immunosuppressive medication; therefore we empirically treated her with ceftriaxone and TMP-SMX alone.

Particularly in the heart transplant population, primary cutaneous nocardiosis remains a rare disease, with our online literature search yielding only three reported cases. ${ }^{5,10,11}$ All the three cases had an identifiable cause: cardiac catheterization ${ }^{5}$, intramuscular injections ${ }^{11}$, and an insect bite. ${ }^{10}$ Our patient had no such identifiable cause, either iatrogenic or traumatic.

In conclusion, nocardiosis is a rare but deadly disease that mainly affects immunocompromised patients. Cutaneous nocardiosis results from traumatic invasion and has three different manifestations, with the primary cutaneous and lymphocutaneous versions common in the developed world. Treatment with TMP-SMX is often overlapped with intravenous antibiotics for serious infections.

\section{References}

1. Filice GA. No0cardiosis. In Fauci AS, Braunwald E, Kasper DL, et al. Harrison's Principles of Internal Medicine. 17 $7^{\text {th }}$ ed. New York, McGraw-Hill Professional, 2008.

2. Schlaberg R, Huard RC, Della-Latta P. Nocardia cyriacigeorgica, an emerging pathogen in the United States. J Clin Microbiol. 2008 Jan;46(1):265-73.

3. Filice GA. Nocardiosis in persons with human immunodeficiency virus infection transplant recipients, and large, geographically defined populations. J Lab Clin Med. 2005 Mar;145(3):156-62.

4. Brown-Elliott BA, Brown JM, Conville PS, et al. Clinical and laboratory features of the Nocardia spp. based on current molecular taxonomy. Clin Microbiol Rev. 2006 Apr;19(2):259-82.

5. Stamenkovic SA, Madden BP. Nocardia asteroides abscess after heart transplantation. J Heart Lung Transplant. 2001 Jul;20(7):789-91.

6. Inamadar AC, Palit A. Primary cutaneous nocardiosis: a case study and review. Indian J Dermatol Venereol Leprol. 2003 Nov-Dec;69(6):386-91.

7. Fukuda H, Saotome A, Usami N, et al. Lymphocutaneous type of nocardiosis caused by Nocardia brasiliensis: a case report and review of primary cutaneous nocardiosis caused by N. brasiliensis reported in Japan. J Dermatol. 2008 Jun;35(6):346-53. Review.

8. Smego RA Jr, Castiglia M, Asperilla MO. Lymphocutaneous syndrome. A review of non-sporothrix causes. Medicine (Baltimore). 1999 Jan;78(1):38-63.

9. Uhde KB, Pathak S, McCullum Jr I, et al Antimicrobial-Resistant Nocardia Isolates, United States, 1995-2004.,Clin Infect Dis. 2010 Nov 8

10. Merigou D, Beylot-Barry M, Ly S, et al. Primary cutaneous Nocardia asteroides infection after heart transplantation. Dermatology. 1998;196(2):246-7.

11. Rees W, Schüler S, Hummel M, et al. Primary cutaneous Nocardia farcinica infection after heart transplantation: a case report. J Thorac Cardiovasc Surg. 1995 Jan;109(1):181-3. 